\title{
Usability of User Interfaces: From Monomodal to Multimodal
}

\author{
Silvia Abrahão ${ }^{1,2}$ \\ 1 Departament de Sistemas Informàtics i Compu- \\ tación, Universidad Politècnica de València \\ Camí de Vera s/n - 46022 València (Spain) \\ 34-96 3877350 \\ sabrahao@dsic.upv.es, \\ abrahao@isys.ucl.ac.be
}

\author{
Jean Vanderdonckt ${ }^{2}$ \\ ${ }^{2}$ Belgian Lab. of Computer-Human Interaction (BCHI), \\ Louvain School of Management (IAG), Université ca- \\ tholique de Louvain, \\ Place des Doyens, 1 - B-1348 Louvain-la-Neuve \\ (Belgium) \\ +32 10/478525 \\ jean.vanderdonckt@uclouvain.be
}

\begin{abstract}
This workshop is aimed at reviewing and comparing existing Usability Evaluation Methods (UEMs) which are applicable to monomodal and multimodal applications, whether they are web-oriented or not. It addresses the problem on how to assess the usability of monomodal user interfaces according to techniques involving one or several modalities, in parallel or combined. In particular, how to synchronize results provided by different UEMs producing various types of results (e.g., audio, video, text, log files) is concerned. It also addresses the problem on how to assess the usability of multimodal user interfaces according to techniques based on multiple modalities. In particular, the question of generalizing the applicability of existing UEMs to these new types of user interfaces is concerned.
\end{abstract}

\section{Categories and Subject Descriptors}

D.2.2 [Software Engineering]: Design Tools and Techniques Computer-aided software engineering (CASE), Evolutionary prototyping, Structured Programming, User Interfaces. H.5.2 [Information Interfaces and Presentation (e.g., HCI)]: User interfaces - Graphical user interfaces, Interaction styles, Input devices and strategies, Prototyping, Voice I/O.

\section{General Terms}

Measurement, Performance, Design, Experimentation, Human Factors, Standardization, Languages.

\section{Keywords}

Accessibility, Automated evaluation, Monomodal applications, Multimodal user interfaces, Multimodal web interfaces, Usability engineering, Usability evaluation method, Usability testing, Usability guidelines, Web engineering.

\section{MOTIVATIONS}

Today, existing applications tend to shift their locus of interaction from the graphic channel to other channels such as speech, gesture, and haptic, to name a few. For instance, new markup languages exist today for developing multimodal web applica-

(c), Silvia Abrahão \& Jean Vanderdonckt, 2007 Published by the British Computer Society Volume 2 Proceedings of the 21 st BCS HCI Group Conference HCI 2007, 3-7 September 2007, Lancaster University, UK Devina Ramduny-Ellis \& Dorothy Rachovides (Editors) tions, such as VoiceXML, $\mathrm{X}+\mathrm{V}, \mathrm{SVG}$. The W3C Multimodal Interaction Framework offers multiple ways of implementing multimodal web applications, also leaving several degrees of freedom to the designer and the developer. This new locus of interaction poses unprecedented challenges for assessing the usability of such applications. It is not because we are able to technically develop these multimodal user interfaces that we can guarantee their usability. Existing usability evaluation methods (UEMs) which mainly consider the graphic channel cannot be directly reused for other modalities of interaction. Moreover, UEMs which are applicable for one modality only (e.g., speech) may become inappropriate for applications combining several modalities (e.g., speech and haptic). In the other way around, UEMs which are particularly suited for one modality may become of some interest for other channels if they bring some new ideas on how to assess the usability. For instance, eye tracking techniques may be used to detect the visual paths of a user on a screen, a web page, even if eye tracking is not used as an input modality.

The motto of this workshop is that we need to evaluate multimodal user interfaces as a whole and not as the sum of pieces involving a combination of individual interaction modalities. Therefore, this workshop is intended to examine existing UEMs for individual modalities (e.g., graphic, speech) as well as for combined modalities. This does not mean that it should be restricted to multimodal applications only: UEMs valid for monomodal applications would be also very interesting for being transferred to the multimodal domain. Therefore, monomodal or multimodal UEMs would be considered for monomodal and multimodal applications, whether they are intended for the web or not.

\section{TOPICS OF INTEREST}

In this one-day workshop, we invite contributions, which discuss methodological, technical, application-oriented and theoretical aspects of the usability evaluation of monomodal and multimodal user interfaces. These topics include, but are not limited to:

- Adaptation and identification of ergonomic/HCI criteria and principles to multimodal interfaces

- Application of any existing UEM or modified one to one or several case studies recommended by the workshop

- Classification of usability models, methods, notations, and tools

- Evaluation of user's performance in a multimodal context of use

- Experimental studies conducted on multimodal interfaces

- Experimentation with cognitive models of user interaction for multimodal interfaces 
- Tools for automatic or computer-aided usability evaluation of multimodal interfaces

- Tools for capturing usability knowledge for monomodal and multimodal

- Usability and accessibility guidelines for monomodal and multimodal interfaces

- Usability evaluation method for monomodal and multimodal interfaces

- Usability evaluation of multimodal web interfaces (e.g., speech and gestures)

- Usability factors, criteria, metrics, rules, recommendations

- User experience in multimodal dialogue systems

- Validity of models

\section{METHODOLOGY OF WORK}

Prior to the workshop, a first draft of the white paper will be distributed as a working document to be discussed and expanded during and after the workshop. Based on papers accepted for the workshop and existing experience, this document will discuss a matrix comparing models, methods, notations, and tools existing in the field. Second, participants will be encouraged to apply partially or totally one of their UEM to one or many of the 3 case studies recommended for the workshop. It is expected that by comparing the results provided by different methods on the same case study, significant similarities and differences will emerge. Based on a questionnaire to be filled by workshop participants prior to the workshop, the document will raise significant questions to be addressed by researchers and practitioners belonging to all communities. Discussion groups will be organized around key questions and topics that arise from the accepted papers. It is hoped that these groups can be multidisciplinary, including designers, developers and usability experts.

\subsection{Format}

The first part of the workshop will be dedicated to the presentation of some selected papers accepted for the workshop along with their results on the 3 case studies and limited discussion. The second part will be devoted to a discussion in sub-groups and a plenary session to complete the matrix to be obtained during this workshop. Given our outcomes, we need to use the first part for participants to present their individual understanding of the research problems in this area. The second part will be used to pull together these individual insights into a common framework and to update the first draft of the white paper.

\subsection{Potential participants}

Ideally, 15 to 20 participants will take part in the workshop. All the participants will be asked to submit a 8 pages position paper (maximum) or a 14 pages full paper (maximum). We will encourage papers that address the aforementioned challenges, that present any aspects of a UEM for a monomodal or multimodal interface. We will particularly appreciate for acceptation papers attempting to use their UEM on one or several of the three case studies recommended by the workshop:

1. A multimodal conversational agent, coming from the eNTERFACE'06 workshop (Similar).

2. A multimodal navigation into 3D medical images developed on top of the OpenInterface platform.

3. A multimodal game with two players, one being deaf the other one being mute, developed at eNTERFACE'06 under the lead of Dimitrios Tzovaras (Univ. of Tessaloniki).

These three case studies will be delivered in a packaged form to be downloaded from the workshop web site.

\subsection{Submission procedure}

Authors of papers must submit their papers themselves by APRIL 15th, 2007. All submissions must follow the Journal of Multimodal User Interfaces format (JMUI - http://www.open interface.org/JMUI/) and be submitted electronically in PDF format to the workshop co-chairs at iwumui@similar.cc. All submissions must be maximum 15 (fifteen) pages according to this format. Authors are requested to prepare submissions as close as possible to final camera-ready versions. The submission should clearly emphasize the discussion aspects relevant to the workshop. Members of an international program committee will review all submissions. For the rigorousness of the reviewing process, authors may also submit additional material such as screen dumps, images (e.g. PNG files), videos (e.g., MPEG, AVI files), demonstrations (e.g., Camtasia, SnagIt, Lotus ScreenCam) of software. Some instructions will be put on-line for this purpose. If accepted, this material can also be published on the web site upon agreement of the authors. For questions and comments, please contact the workshop co-chairs at iwumui@similar.cc.

\subsection{Publication}

All papers accepted for the workshop will be first published in the workshop proceedings. Provided that accepted papers are substantive both in quantity and quality, a special issue of the Journal of Multimodal User Interfaces (JMUI - http://www. openinterface.org/JMUI/) has been already agreed. The white paper that will be edited by the workshop co-chairs will be the introducing paper of this special issue. The description of the implementation of the three case studies will then be provided in appendix.

\section{ACKNOWLEDGMENTS}

The workshop is mainly sponsored by the European COST Action ${ }^{\circ} 294$ MAUSE (Towards the Maturation of IT Usability Evaluation, www.cost294.org) and by SIMILAR, the European research task force creating human-machine interfaces similar to human-human communication (http://www.similar.cc). Several members of this network of excellence and of this COST action are members of the above Program Committee and guarantee a large geographical and topical coverage of the workshop. It is also supported by the OpenInterface Foundation (www.openinterface.org) supported by FP6-IST4 and the UsiXML Consortium (www.usixml.org).

\section{REFERENCES}

[1] Law, E., Hvannberg, E., Cockton, G., Palanque, Ph., Scapin, D., Springett, M., Stary, Ch., and Vanderdonckt, J. Towards the Maturation of IT Usability Evaluation (MAUSE). In Proc. of $10^{\text {th }}$ IFIP TC 13 Int. Conf. on $\mathrm{Hu}$ man-Computer Interaction INTERACT'2005 (Rome, 1216 September 2005). Lecture Notes in Computer Science, Vol. 3585, Springer-Verlag, Berlin, 2005, pp. 1134-1137.

[2] Law, E., Hvannberg, E., and Cockton, G. (eds.). Maturing Usability: Quality in Software, Interaction and Value. HCI Series, Springer-Verlag, Berlin, 2007.

[3] Mariage, C., Vanderdonckt, J., Pribeanu, C. State of the Art of Web Usability Guidelines. In "The Handbook of Human Factors in Web Design", Proctor, R.W., Vu, K.Ph.L. (eds.), Chapter 21. Lawrence Erlbaum Associates, Mahwah, 2005, pp. 688-700.

[4] Vanderdonckt, J. Development Milestones towards a Tool for Working with Guidelines. Interacting with Computers 12, 2 (December 1999) 81-118. 